\title{
Suppl. Information:
}

Table 1. Primer sequences used for qPCR:

\begin{tabular}{|c|c|c|}
\hline Primer & Sequence & Orientation \\
\hline \multirow[t]{2}{*}{$h W N T 10 B$} & 5’-TGG GAT GTG TAG CCT TCT CC-3' & $\mathrm{S}$ \\
\hline & 5'-CCC AGC CAA AAG GAG TAT GA-3' & AS \\
\hline \multirow[t]{2}{*}{$h C D K 4$} & 5’-TCA TGC CAA TTG CAT CGT TCA CCG -3' & $\mathrm{S}$ \\
\hline & 5’-ACA GGT GTT GCA TAT GTG GAC TGC -3' & AS \\
\hline \multirow[t]{2}{*}{$h C C N D 1$} & 5’-CAG AAG TGC GAA GAG GAG GTC-3’ & S \\
\hline & 5'-TCA TCT TAG AGG CCA CGA ACA T-3' & AS \\
\hline \multirow[t]{2}{*}{ hCCNE1 } & 5’-TTA CCC AAA CTC AAC GTG CAA GCC-3' & $\mathrm{S}$ \\
\hline & 5’- AGA GGG TGT TGC TCA AGA AAG TGC-3’ & AS \\
\hline \multirow[t]{2}{*}{ hCCNA1 } & 5’- TGT CTG TTC TGA GAG GGA AAC TGC -3’ & $\mathrm{S}$ \\
\hline & 5’- AAG GAG AAA CTG GTT GGT GGT TGG -3’ & AS \\
\hline \multirow[t]{2}{*}{ hCCNB1 } & 5’- ATT GTG TGC CCA AGA AGA TGC TGC -3’ & $\mathrm{S}$ \\
\hline & 5’- TTA GAT GCT CTC CGA AGG AAG TGC-3’ & AS \\
\hline \multirow[t]{2}{*}{ hBIRC5 } & 5’- CCG CAT CTC TAC ATT CAA GAA CTG GC & S \\
\hline & 5’- TTG ACA GAA AGG AAA GCG CAA CCG - & AS \\
\hline \multirow[t]{2}{*}{ hAXIN2 } & 5’-TCA AGT GCA AAC TTT CGC CAA CCG-3’ & $\mathrm{S}$ \\
\hline & 5’-TGG TGC AAA GAC ATA GCC AGA ACC-3’ & AS \\
\hline \multirow[t]{2}{*}{$h M Y C$} & 5’-TCT CCA CAC ATC AGC ACA ACT ACG-3' & $\mathrm{S}$ \\
\hline & 5’-TGT GTT CGC CTC TTG ACA TTC TCC-3’ & AS \\
\hline \multirow[t]{2}{*}{ hHMGA2 } & 5'-TCCGTGCCCGACCCTAT-3' & $\mathrm{S}$ \\
\hline & 5’-CCTTGAAATGTTAGGCGGGG -3' & AS \\
\hline \multirow[t]{2}{*}{ hPCNA } & 5’- TGT AAA CCT GCA GAG CAT GGA CTC G- & $\mathrm{S}$ \\
\hline & 5’- AAA TAC TAG CGC CAA GGT ATC CGC-3’ & AS \\
\hline \multirow[t]{2}{*}{$h S L U G$} & 5’- TTT CTG GGC TGG CCA AAC ATA AGC -3’ & $\mathrm{S}$ \\
\hline & 5’- AAT GTG TCC TTG AAG CAA CCA GGG -3’ & AS \\
\hline \multirow[t]{2}{*}{ hFIBRONECTIN } & 5’- TGT CAA CGA AGG CTT GAA CCA ACC -3’ & $\mathrm{S}$ \\
\hline & 5’- AGT TCA CAC CAT TGT CAT GGC ACC -3’ & AS \\
\hline \multirow[t]{2}{*}{ hVIMENTIN } & 5’-ATG AAA GTG TGG CTG CCA AGA ACC-3’' & $\mathrm{S}$ \\
\hline & 5’-TCT CTT CCA TTT CAC GCA TCT GGC-3’ & AS \\
\hline \multirow[t]{2}{*}{ hZEB1 } & 5’-ATG CAC AAC CAA GTG CAG AAG AGC-3' & $\mathrm{S}$ \\
\hline & 5’-AGG CTG ATC ATT GTT CTT GGC AGG-3’ & AS \\
\hline \multirow[t]{2}{*}{ h18S } & 5’- CCG CGG TTC TAT TTT GTT GGT -3’ & $\mathrm{S}$ \\
\hline & 5’- CTC TAG CGG CGC AAT ACG A -3' & AS \\
\hline
\end{tabular}

Legend: Antisense (AS), Sense (S), and human (h). 